\title{
Perfil de lípidos y ácidos grasos de Perna viridis, mejillón verde (Mollusca: Bivalvia) en el oriente venezolano y la costa oeste de Trinidad
}

\author{
Tamar Koftayan ${ }^{1 *}$, Jahiro Milano ${ }^{2}$, Haydelba D' Armas $^{3}$ \& Gabriel Salazar ${ }^{4}$ \\ 1. Universidad de Oriente, Núcleo de Monagas, Maturín, estado Monagas 1601, Venezuela; tkoftayan@yahoo.com \\ 2. Universidad de Oriente, Núcleo de Bolívar, Instituto Limnológico, Caicara del Orinoco 1601, estado Bolívar, \\ Venezuela;jjmg43@yahoo.es \\ 3. Universidad de Oriente, Núcleo de Sucre, Departamento de Química, Cumaná, estado Sucre 1601, Venezuela; \\ haydelba@yahoo.com \\ 4. Instituto Universitario de Tecnología de Cumaná, estado Sucre 1601, Venezuela; gesalazar@cantv.net \\ * Correspondencia
}

Recibido 28-VI-2010. Corregido 20-IX-2010. Aceptado 21-X-2010.

\begin{abstract}
Lipid and fatty acid profile of Perna viridis, Green Mussel (Mollusca: Bivalvia) in different areas of the Eastern Venezuela and the West coast of Trinidad. The species Perna viridis is a highly consumed species, which fast growth makes it an interesting aquaculture alternative for Venezuelan and Trinidad coasts. With the aim to contribute with its nutritional value information, this study analyzed lipid and fatty acid contents from samples taken in five locations from Eastern Venezuela and three from Trinidad West Coast. Total lipids were extracted and quantified, from a pooled sample of 100 organisms per location, by standard gravimetric methods, and their identification and quantification was done by TLC/FID (Iatroscan system). Furthermore, the esterified fatty acids of total lipid, phospholipids and triacylglycerols were identified and quantified by gas chromatography. Eastern Venezuela samples from Los Cedros, La Brea and Chaguaramas showed the highest total lipid values of 7.92, 7.74 and 7.53, respectively, and the minimum values were obtained for La Restinga (6.08\%). Among lipid composition, Chacopata samples showed the lowest phospholipid concentration (48.86\%) and the maximum values for cholesterol (38.87\%) and triacylglycerols (12.26\%); besides, La Esmeralda and Rio Caribe samples exhibited maximum phospholipids ( 88.71 and 84.93 respectively) and minimum cholesterol (6.50 and $4.42 \%$ ) concentrations. Saturated fatty acids represented between $15.04 \%$ and $65.55 \%$ within total lipid extracts, with maximum and minimum values for La Esmeralda and Chacopata, respectively. Polyunsaturated results resulted between 7.80 and 37.18\%, with higher values in La Brea and lower values in La Esmeralda. For phospholipids, saturated fatty acids concentrations varied between 38.81 and $48.68 \%$ for Chaguaramas and Chacopata samples, respectively. In the case of polyunsaturated fatty acids, these varied between non detected and $34.51 \%$, with high concentrations in Los Cedros (27.97\%) and Chaguaramas (34.51\%) samples. For the triacylglycerols, the saturated fatty acids composition oscillated between 14.27 and $53.80 \%$ with low concentrations for Chacopata and high concentration for La Restinga; the polyunsaturated fatty acids were between 4.66 and $35.55 \%$ with lower values for Chacopata and higher values for Chaguaramas samples. P. viridis is recommended for human being consumption, according to the high content of unsaturated fatty acids found for this species. Rev. Biol. Trop. 59 (1): 171-182. Epub 2011 March 01.
\end{abstract}

Key words: bivalve, fatty acids, lipids, triaciylglycerol, Perna viridi.

Perna viridis es un mejillón que mide de 80 a $100 \mathrm{~mm}$ de largo. Es una especie filtradora que se alimenta, principalmente, de detritos, zooplancton y fitoplancton. Tiene sexos separados y su fertilización es externa. Esta especie tiene tres desoves al año: noviembrediciembre, julio-agosto (mayor intensidad) y de febrero a abril. Este bivalvo habita principalmente en sistemas estuarinos, donde los rangos de salinidad son de 18 a $33 \%$ y temperatura 
de 11 a $32^{\circ} \mathrm{C}$. Actualmente, esta especie es muy resistente a los cambios de temperatura y salinidad, tolerando niveles de salinidad de 0 a $80 \%$ y temperaturas de 7 a $37.5^{\circ} \mathrm{C}$ (Vélez \& Martínez 1967, García 2003).

En Venezuela, se han realizado algunos estudios sobre el género Perna, recientemente, se caracterizó y cuantificó el contenido de ácidos grasos presente en las gónadas y músculo de la especie de mejillón $P$. perna, y se encontró que los ácidos grasos para los estadíos II Y III presentó un porcentaje de ácidos grasos poliinsaturados mayor que el de los saturados y monoinsaturados (Malavé 2002). También se realizaron dos trabajos en los que se analizaron las toxinas presentes en las ostras y mejillones provenientes de Venezuela y Trinidad, en los cuales se demostró que las ostras de Yaguaraparo contienen toxinas diferentes a las de Pedernales; de igual manera, las toxinas encontradas en los mejillones de Güiria son diferentes a las de Chaguaramas, mientras que en La Brea y Los Cedros se hallaron toxinas similares. Los niveles de toxinas PSP no exceden el límite máximo permisible (MPL) en los bivalvos de Venezuela estudiados, sin embargo, en Trinidad viola el MPL en los mejillones.

Tanto en Venezuela como en Trinidad, $P$. viridis es altamente consumida por las personas que habitan en las zonas costeras; sin embargo, no existen trabajos previos sobre la composición de ácidos grasos en ninguno de estos países. Estos bivalvos, son una fuente rica en lípidos y constituyen una fuente importante de energía metabólica para mantener la estructura e integridad de las membranas celulares; pero a pesar de su gran importancia, se han realizado pocos estudios sobre el contenido lipídico en estos organismos marinos (Cowey \& Sargent 1988).

La proporción de lípidos y ácidos grasos en el cuerpo de los moluscos, está íntimamente relacionado con el estado fisiológico del organismo, el crecimiento, dieta alimenticia, reacciones del organismo ante las variaciones del medio y en general, con todos los factores internos y externos que inciden en un momento dado en la vida de cada individuo (Maza 1991).
Por lo expuesto anteriormente, se realizó una evaluación comparativa de la composición lipídica y de ácidos grasos de $P$. viridis, recolectada en distintos lugares del oriente venezolano y la costa oeste de Trinidad (en vista de su situación geográfica en el Golfo de Paria), con el fin de determinar su valor nutricional y energético en las localidades: La Esmeralda, Río Caribe, Chacopata y la Laguna de la Restinga, pertenecientes a Venezuela y en Trinidad: Los Cedros, La Brea y Chaguaramas, e informar a la población interesada acerca de las propiedades nutricionales que ofrece su ingesta $y$ contribuir con el desarrollo de la acuicultura de la especie, contribuyendo con el sustento económico y alimenticio de los seres humanos de las zonas de estudio, por ser especies prolíficas.

\section{MATERIALES Y MÉTODOS}

Áreas de estudio: La recolección de las muestras se realizó en cuatro zonas del oriente venezolano: Chacopata, ubicada al norte de la Península de Araya (Estado Sucre), frente a la isla de Los Lobos a los $63^{\circ} 52^{\prime} \mathrm{O}-10^{\circ} 42^{\prime}$ $\mathrm{N}$; Laguna de La Restinga (constituido por una estrecha franja de arena, que une la parte oriental de la isla de Margarita con la Península de Macanao, a $10^{\circ} 54^{\prime} \mathrm{N}-64^{\circ} 45^{\prime} \mathrm{O}$ ); Río Caribe (situada en el litoral norte de la península de Paria en el mar Caribe, que está a $10^{\circ} 38^{\prime}$ $\mathrm{N}$ - longitud $63^{\circ} 0^{\prime} \mathrm{O}$; La Esmeralda (ubicada al norte de la Península de Paria en el mar Caribe). Y tres zonas de la Isla de Trinidad: Chaguaramas (Península que se extiende por el territorio noroccidental de Trinidad, la mayor isla de la República insular de Trinidad y Tobago $\left(10^{\circ} 02^{\prime} \mathrm{N}-61^{\circ} 37^{\prime} \mathrm{O}\right)$; La Brea (Está localizada en la costa sur oeste de Trinidad $\left(10^{\circ} 14^{\prime}\right.$ $\mathrm{N}-61^{\circ} 37^{\prime} \mathrm{O}$ ); Los Cedros (ubicada al sur de Trinidad, $\left.10^{\circ} 05^{\prime} \mathrm{N}-61^{\circ} 51^{\prime} \mathrm{O}\right)$ (Rojas 2002).

Recolección de muestras: Los mejillones fueron recolectadas en las lagunas de La Restinga, Chacopata, La Esmeralda y Río Caribe (Venezuela); y Los Cedros, Chaguaramas y La Brea (Trinidad), durante los meses de junio y julio de 2001 (desove de la especie y presentan 
la mayor talla comercial). En cada área de estudio se recolectaron pools de 100 mejillones con tallas de 85 a $90 \mathrm{~cm}$. Se transportaron al laboratorio en una cava con hielo seco. Se les extrajo el tejido blando, que se limpió y lavó con agua desionizada para eliminar la mayor cantidad de sales, iones y fitoplancton presentes.

Obtención de los extractos lipídicos: A cada muestra se le extrajeron los lípidos totales, tomando porciones de $1 \mathrm{~g}$ de muestra por $20 \mathrm{ml}$ de cloroformo:metanol (2:1), para cada localidad. Este procedimiento garantizó la extracción completa de los lípidos. Los análisis se realizaron seis veces por muestras. Se determinó el porcentaje de lípidos en las muestras por gravimetría (Overturf \& Dryer 1967, Carter 1993).

Caracterización y cuantificación de los lípidos utilizando Iatroscan: Una porción del extracto de los lípidos se analizó por cromatografía de capa fina automatizada (TLC-FID). Los análisis se realizaron por triplicados y se llevaron a cabo en un analizador Iatroscan TH-10 TLC-FID operado con un Iatrocorder TC-11 como integrador. El detector de ionización en llama se operó a un flujo de aire de $0.6 \mathrm{ml} / \mathrm{min}$ (bomba generadora) y un flujo de hidrógeno de $190 \mathrm{ml} / \mathrm{min}$. La velocidad de análisis de flujo fue $30 \mathrm{~cm} / \mathrm{min}$ (Ackman 1982).

Los cromatogramas se desarrollaron en un tanque cromatográfico con una mezcla de hexano:éter dietílico (60:40), una vez corrido el disolvente, las varillas se secaron a $100^{\circ} \mathrm{C}$ aproximadamente por tres minutos en un horno Iatron-Rod Dryer e, inmediatamente, se analizaron con el Iatroscan. La identificación de los lípidos presentes en el extracto, se realizó por comparación de los tiempos de retención con los registrados para patrones comerciales. El contenido lipídico se expresó como un porcentaje de los lípidos identificados.

Aislamiento de los triacilgliceroles y fosfolípidos: Los triacilgliceroles y fosfolípidos presentes en los extractos de los lípidos totales, se separaron mediante el uso de cromatografía de capa fina preparativa (CCFP). Para tal fin, se utilizó como adsorbente sílica gel en capas de $1.0 \mathrm{~mm}$ de espesor sobre placas $20 \times 20 \mathrm{~cm}^{2}$ y como solvente eluyente una mezcla de éter de petróleo: éter dietílico: ácido acético (87:12:1). Un primer desarrollo cromatográfico se realizó con estándares de lípidos conocidos de Sigma Chemical Company y se determinaron sus $\mathrm{R}_{\mathrm{f}}$; los cuales se utilizaron como patrones para separar e identificar los lípidos en las muestras de mejillones; que se identificaron por comparación de los $\mathrm{R}_{\mathrm{f}}$ de las bandas obtenidas con las de los patrones comerciales. Una vez que los triacilgliceroles y fosfolípidos se separaron de las placas, se extrajeron con éter dietílico y metanol, respectivamente (Milano 2003).

Caracterización y cuantificación de ácidos grasos: Se realizó la conversión de los ácidos grasos presentes en los lípidos totales, fosfolípidos y triacilgliceroles a ésteres metílicos (Coristie 1973). La composición porcentual de los ácidos grasos en los extractos de lípidos totales, triacilgliceroles y fosfolípidos, se determinó mediante la cromatografía de gas-líquido. Los ésteres metílicos correspondientes a cada muestra se analizaron en un cromatógrafo de gas-líquido Agilent modelo 4890 D, acoplado a un detector de ionización de llama (FID) y una columna HP-INNOXWAX 30m $0.32 \mathrm{~mm} 1 \mathrm{D}$, fase líquida. Como gas de arrastre se utilizó nitrógeno a un flujo de $1.8 \mathrm{ml} / \mathrm{min}$. La separación se realizó en las siguientes condiciones: temperatura del inyector y el detector de $300^{\circ} \mathrm{C}$ y de la columna $200^{\circ} \mathrm{C}$.

El área de los picos se determinó con un sistema de adquisición de data HP3398AGC CHEMSTATION acoplado al cromatógrafo. La identificación de ácidos grasos se realizó mediante la comparación de los tiempos de retención, determinados con los patrones comerciales de ésteres metílicos (Farnworth et al. 1982). Los resultados se expresaron como un porcentaje de la masa total de los ácidos grasos en los extractos de lípidos totales, triacilgliceroles y fosfolípidos, para las muestra de mejillones en cada localidad.

A los resultados de los lípidos totales y las diferentes clases de lípidos en los mejillones 
de las localidades, se le aplicó un análisis de varianza de un factor. En los casos donde se determinaron diferencias significativas $(\mathrm{p}<0.05)$ se aplicó la prueba a posteriori de Duncan. Los datos fueron analizados utilizando un paquete estadístico Statgraphics plus 4.1.

\section{RESULTADOS}

Análisis de los lípidos: Las mayores concentraciones de lípidos se presentan en las muestras de Los Cedros, La Brea y Chaguaramas con valores de 7.92, 7.74 y 7.53; respectivamente (Cuadro 1). El análisis de varianza indicó que existen diferencias significativas $(\mathrm{p}<0.05)$ entre las muestras estudiadas. La prueba a posteriori de Duncan mostró la formación de tres grupos: el primer grupo homogéneo integrado por las muestras de las localidades de La Restinga, La Esmeralda y Río Caribe, el segundo grupo homogéneo por las de La Esmeralda, Río Caribe y Chacopata, y el tercero por las de Chaguaramas, La Brea y Los Cedros.

Análisis de lípidos: En las localidades de Venezuela y Trinidad dominaron los fosfolípidos con valores máximos en las muestras de La Esmeralda $(88.71 \%)$ y Río Caribe $(84.93 \%)$. Estas áreas se encuentran en condiciones fluviales similares con aguas relativamente saladas (36.88\%o) en comparación con las otras zonas estudiadas; seguidas por La Restinga (67.07\%), Chaguaramas (62.19\%), Los Cedros (61.59\%), La Brea (60.88\%) y Chacopata $(48.86 \%)$ (Cuadro 2). El colesterol se presenta con valores máximos de concentración de $38.87 \%$ en la muestra de Chacopata, seguido de Los Cedros (33.49\%), Chaguaramas, La Brea, La Restinga y valores mínimos en las muestras de Río Caribe y La Esmeralda con 6.50 y $4.92 \%$, respectivamente. Posteriormente, se encuentran los triacilgliceroles, con valores máximos en las muestras de Chacopata con $12.26 \%$, Río Caribe (8.57\%), La Brea (8.44\%) y valores mínimos en Los Cedros con (4.93\%). Las únicas muestras de mejillones que presentaron ésteres de colesterol fueron los de $\mathrm{La}$ Brea con valores de $2.72 \%$.

El análisis de varianza realizado a los valores de fosfolípidos presentó diferencias altamente significativas $(\mathrm{p}<0.05)$ entre localidades. La prueba a posteriori de Duncan (Cuadro 2) mostró la formación de tres grupos: el primer grupo homogéneo integrado por las muestras de Chacopata, donde se encontraron los valores mínimos de fosfolípidos; el segundo grupo homogéneo formado por La Brea, Los Cedros, Chaguaramas y La Restinga y el tercero por las muestras de Río Caribe y La Esmeralda.

El análisis de varianza realizado a los valores de colesterol encontrado en $P$. viridis,

\section{CUADRO 1}

Porcentajes de lípidos totales de $\mathrm{P}$. viridis provenientes de las diferentes áreas de estudio

TABLE 1

Percentages of $\mathrm{P}$. viridis total lipids from different study areas

\begin{tabular}{|c|c|c|c|c|c|}
\hline Localidades & $\mathrm{N}$ & $\mathrm{X}$ & $\mathrm{S}$ & $\mathrm{Sx}$ & $\mathrm{D}$ \\
\hline La Restinga* Chacopata & 6 & 6.08 & 0.04 & 0.21 & \\
\hline La Esmeralda* & 6 & 6.36 & 0.07 & 0.21 & \\
\hline Río Caribe* & 6 & 6.37 & 0.04 & 0.21 & \\
\hline Chacopata* & 6 & 6.77 & 0.03 & 0.21 & \\
\hline Chaguaramas** & 6 & 7.53 & 0.06 & 0.21 & \\
\hline La Brea** & 6 & 7.74 & 0.27 & 0.21 & \\
\hline Los Cedros*** & 6 & 7.92 & 0.71 & 0.21 & \\
\hline
\end{tabular}

$\mathrm{N}=$ número de réplicas, $\mathrm{X}=$ media, $\mathrm{S}=$ desviación estándar, $\mathrm{Sx}=$ error estándar, $\mathrm{D}=$ Duncan. *Venezuela, **Trinidad. 
CUADRO 2

Concentración de lípidos de $\mathrm{P}$. viridis procedente de las diferentes áreas de estudio

TABLE 2

Lipid concentrations of $\mathrm{P}$. viridis from different study areas

\begin{tabular}{|c|c|c|c|c|c|}
\hline Localidades & $\begin{array}{c}\text { Fosfolípidos } \\
\%(\mathrm{X} \pm \mathrm{S})\end{array}$ & Df & $\begin{array}{c}\text { Colesterol } \\
\%(\mathrm{X} \pm \mathrm{S})\end{array}$ & Dc & $\begin{array}{c}\text { Triacilgliceroles } \\
\%(\mathrm{X} \pm \mathrm{S})\end{array}$ \\
\hline Chacopata* & $48.86 \pm 4.70$ & & $38.87 \pm 6.14$ & & $12.26 \pm 1.44$ \\
\hline La Brea** & $60.88 \pm 4.14$ & & $27.96 \pm 2.28$ & & $8.44 \pm 0.99$ \\
\hline Los Cedros** & $61.59 \pm 2.15$ & & $33.49 \pm 1.18$ & & $4.93 \pm 0.96$ \\
\hline Chaguaramas** & $62.19 \pm 6.16$ & & $33.02 \pm 6.84$ & & $4.79 \pm 0.68$ \\
\hline La Restinga* & $67.07 \pm 0.66$ & & $27.12 \pm 0.52$ & & $5.81 \pm 0.15$ \\
\hline Río Caribe* & $84.93 \pm 1.88$ & & $6.50 \pm 3.17$ & & $8.57 \pm 5.05$ \\
\hline La Esmeralda* & $88.71 \pm 0.04$ & & $4.92 \pm 0.28$ & & $6.37 \pm 0.25$ \\
\hline
\end{tabular}

$\mathrm{N}=$ número de réplicas (03), $\mathrm{X}=$ media, $\mathrm{S}=$ desviación estándar, $\mathrm{Df}=$ Duncan para fosfolípidos, Dc=Duncan para colesterol. *Venezuela; **Trinidad.

el cual presentó diferencias altamente significativas $(\mathrm{p}<0.05)$ entre las muestras de las distintas localidades. La prueba a posteriori de Duncan (Cuadro 2) mostró la formación de tres grupos: el primer grupo homogéneo integrado por la muestras de La Esmeralda y Río Caribe, el segundo grupo homogéneo por La Restinga, La Brea, Chaguaramas y Los Cedros, y el tercero por las de Chaguaramas, Los Cedros y Chacopata.

Los valores de triacilgliceroles fueron mayores en las muestras provenientes de Chacopata (12.26\%). No obstante, el análisis estadístico reveló diferencias no significativas $(\mathrm{p}<0.05)$ entre los mejillones de las distintas áreas de estudio.

\footnotetext{
Análisis de los ácidos grasos en los lípidos totales, fosfolípidos y triacilgliceroles: En el Cuadro 3 se observa la composición de los ácidos grasos en los lípidos totales con altas concentraciones de ácidos grasos no identificados en las muestras estudiadas, entre 13.11 y $54.01 \%$, son presumiblemente en su mayoría ácidos grasos insaturados del tipo poliinsaturado. Los ácidos grasos saturados se encontraron entre 15.04 y $65.55 \%$, con máximos en $\mathrm{La}$ Esmeralda y mínimos en Chacopata, prevaleciendo los ácidos undecanoico (11:0) entre no
}

detectado y $30.90 \%$, con concentraciones mínimas en Chacopata (no detectado) y La Brea (2.51\%), y máximas en Chaguaramas (30.90\%) y Los Cedros (19.93\%); seguidamente del ácido palmítico (16:0) entre un 2.09 y $33.72 \%$, con valores máximos en La Esmeralda y mínimos en Chaguaramas, La Restinga y Chacopata; además de los ácidos mirístico (14:0) y heptadecanoico (17:0) en menores cantidades.

Los ácidos grasos poliinsaturados estuvieron entre un 7.80 y $34.11 \%$, observándose valores marcadamente constantes en las localidades estudiadas, a diferencia de la Esmeralda y Chaguaramas, donde se presentaron valores mínimos entre $10.21 \%$ y $7.80 \%$, respectivamente. Los ácidos grasos que predominaron fueron el adrénico (22:4) entre 7.80 y $25.51 \%$, el octadecatetraenoico (18:4) entre 0.73 y $6.20 \%$, el eicosapentaenoico (20:5 $\omega$-3) y el docosapentaenoico (22:5 $\omega$-3) en menores proporciones.

Los ácidos grasos monoinsaturados son los que están en menores cantidades (entre no detectado y $11.13 \%$ ), siendo notable la diferencia entre los porcentajes totales de ácidos grasos poliinsaturados con respecto a los monoinsaturados, estableciéndose así la relación ácidos poliinsaturados/monoinsaturados.

En el Cuadro 4 se observa la composición porcentual de los ácidos grasos en 
CUADRO 3

Composición porcentual de ácidos grasos de los lípidos totales de $\mathrm{P}$. viridis por área de estudio

TABLE 3

Percentage composition of fatty acids of $\mathrm{P}$. viridis total lipids from the study areas

\begin{tabular}{|c|c|c|c|c|c|c|c|}
\hline Ácido graso & Río Caribe & La Restinga & La Esmeralda & Chacopata & La Brea & Los Cedros & Chaguaramas \\
\hline $11: 0$ & 10.48 & 8.66 & 7.88 & ------ & 2.51 & 19.93 & 30.90 \\
\hline $14: 0$ & 4.44 & 2.91 & 8.58 & 2.30 & 1.71 & 1.85 & 1.14 \\
\hline $16: 0$ & 10.40 & 2.43 & 33.72 & 2.96 & 6.15 & 6.25 & 2.09 \\
\hline $17: 0$ & 1.97 & ----- & 4.53 & ------ & 0.59 & ----- & ------ \\
\hline Total AGS & 35.47 & 35.26 & 65.55 & 15.04 & 37.34 & 40.04 & 41.44 \\
\hline $16: 1$ & 4.39 & ------ & 9.98 & ------ & 1.94 & 1.23 & 1.10 \\
\hline $18: 1$ & 1.07 & ------ & 1.15 & ----- & 1.96 & 0.49 & ----- \\
\hline Total AGMI & 5.46 & ------ & 11.13 & ------ & 3.90 & 1.72 & 1.10 \\
\hline $18: 4$ & 3.93 & 1.82 & 0.73 & 6.20 & 1.52 & 1.50 & ------ \\
\hline $20: 5 \omega-3$ & ------ & ------ & 3.34 & ------ & 2.79 & 1.07 & ------ \\
\hline $22: 4$ & 25.51 & 24.75 & ------ & 25.48 & 22.72 & 17.88 & 7.80 \\
\hline $22: 5 \omega-3$ & ------ & ------ & 2.84 & 0.78 & 2.39 & ------ & ------ \\
\hline Total AGPI & 30.29 & 28.56 & 10.21 & 33.24 & 34.11 & 21.07 & 7.80 \\
\hline Total AGNI & 28.78 & 36.19 & 13.11 & 54.01 & 24.65 & 37.18 & 49.66 \\
\hline
\end{tabular}

$\mathrm{N}=3$, números de réplicas (pools de 60 mejillones); $\mathrm{AG}=$ ácidos grasos saturados; $\mathrm{AGMI=ácidos} \mathrm{grasos} \mathrm{monoinsaturados;}$ AGPI = ácidos grasos poliinsaturados, AGNI=ácidos grasos no identificados; ------- = no detectado; Valores encontrados $=($ medias \pm 0.1$)$; Límite de detección $\geq 0.1$.

los fosfolípidos. Se puede observar las altas concentraciones de ácidos grasos no identificados en las muestras estudiadas, entre 20.60 y $59.37 \%$, los cuales se presume que son en su mayoría ácidos grasos insaturados del tipo poliinsaturados. La concentración de ácidos grasos saturados es similar en las distintas localidades, encontrándose entre 38.81 y $48.68 \%$ con mínimos en las muestras de Chaguaramas (38.81\%) y La Esmeralda (39.95\%), y máximos en Chacopata (48.68) y Río Caribe (48.23\%), con un predominio del ácido undecanoico (11:0) entre no detectado y $35.16 \%$, este ácido no fue detectado en Los Cedros y estuvo presente con un $0.79 \%$ en Chaguaramas, a pesar de presentar altas concentraciones en las demás localidades; seguidamente los ácidos palmítico (16:0) y mirístico (14:0).

Los ácidos grasos poliinsaturados se encontraron entre no detectado y $34.51 \%$, con máximas concentraciones en las localidades de Los Cedros (27.97\%) y Chaguaramas
(34.51\%). Los ácidos grasos prevalecientes fueron el octadecatetraenoico (18:4) con $8.37 \%$ en las muestras de Chaguaramas; el octadecatrienoico (18:3) con máximas de $1.32 \%$ en la localidad de Rio Caribe y en los Cedros y Chaguaramas dominaron el adrénico (22:4) con 7.31 y $7.08 \%$, el docosapentaenoico (22:5 $\omega-3)$ con 8.95 y $2.18 \%$, el docosahexaenoico (22:6 $\omega-3)$ con 1.73 y $7.38 \%$ y el araquidónico (20:4) con 5.88 y $1.80 \%$, respectivamente. Los ácidos grasos monoinsaturados se encontraron en menor proporción en todas las muestras en estudio, donde los valores oscilaron entre no detectado y $6.08 \%$.

En la Cuadro 5 se observa que la composición de ácidos grasos saturados presentes en los triacilgliceroles de las muestras en estudio variaron entre 14.27 y $53.80 \%$, con mínimos en Chacopata y máximos en La Restinga; prevaleciendo los ácidos palmítico (16:0), cuyos valores oscilaron entre no detectado y $14.67 \%$, mirístico (14:0) entre no detectado y $7.89 \%$, 
CUADRO 4

Composición porcentual de ácidos grasos de los fosfolípidos de $\mathrm{P}$. viridis por área de estudio

TABLE 4

Percentage composition of fatty acids of $\mathrm{P}$. viridis phospholipids from the study areas

\begin{tabular}{|c|c|c|c|c|c|c|c|}
\hline Ácido graso & Río Caribe & La Restinga & La Esmeralda & Chacopata & La Brea & Los Cedros & Chaguaramas \\
\hline 11:0 & 21.70 & 35.16 & 31.18 & 34.84 & 34.34 & ------ & 0.79 \\
\hline $14: 0$ & 5.37 & 0.63 & 0.98 & 2,54 & ------ & 0,76 & 4,38 \\
\hline $16: 0$ & 6.25 & 0.95 & 1.95 & 3.69 & 0.37 & 1.22 & 5.98 \\
\hline $17: 0$ & 2.46 & 0.70 & 1.19 & 0.67 & 0.42 & ------ & 4.56 \\
\hline Total AGS & 48.23 & 44.81 & 39.95 & 48.68 & 44.03 & 44.29 & 38.81 \\
\hline $16: 1$ & ------ & 0.52 & 0.68 & 1.24 & ------ & ----- & 2.67 \\
\hline $18: 1$ & 1.56 & ------ & ------ & ------ & ----- & 0.70 & 2.55 \\
\hline Total AGMI & 3.40 & 0.52 & 0.68 & 1.24 & ------ & 1.20 & 6.08 \\
\hline $18: 3$ & 1.32 & 0.24 & ------ & 0.25 & ------ & ----- & ------ \\
\hline $18: 4$ & 1.94 & 0.27 & ------ & 0.14 & ----- & 3.01 & 8.37 \\
\hline $20: 4$ & ------ & ------ & ------ & 0.38 & ------ & 5.88 & 1.80 \\
\hline $20: 5 \omega-3$ & ----- & ----- & ------ & 1.15 & ----- & ------ & 7.80 \\
\hline $22: 4$ & 5.42 & ------ & ------ & ------ & ------ & 7.31 & 7.08 \\
\hline $22: 5 \omega-3$ & ------ & ----- & ------ & ------ & ------ & 8.95 & 2.18 \\
\hline $22: 6 \omega-3$ & ------ & ----- & ----- & ----- & ------ & 1.73 & 7.38 \\
\hline Total AGPI & 11.15 & 0.51 & ------ & 1.92 & ------ & 27.97 & 34.51 \\
\hline Total AGNI & 37.22 & 54.17 & 59.37 & 48.16 & 55.97 & 26.54 & 20.60 \\
\hline
\end{tabular}

$\mathrm{N}=3$, números de réplicas (pools de 60 mejillones); AGS=ácidos grasos saturados; AGMI=ácidos grasos monoinsaturados; AGPI=ácidos grasos poliinsaturados; AGNI=ácidos grasos no identificados; ------- = no detectado; Valores encontrados $=($ medias \pm 0.1$)$; Límite de detección $\geq 0.1$.

y heptadecanoico (17:0) entre no detectado y $7.80 \%$.

Los ácidos grasos poliinsaturados estuvieron presentes entre 4.66 y $35.55 \%$, con mínimos en las muestras de Chacopata (4.66\%) y La Brea (14.10\%), y máximos en Chaguaramas. El ácido graso más relevante fue el adrénico (22:4), el cual varió entre no detectado y $23.33 \%$; seguidamente de los ácidos octadecatetraenoico (18:4) y octadecatrienoico (18:3); también se presentaron los ácidos eicosapentaenoico (20:5) y docosapentaenoico (22:5) de la familia $\omega-3$.

Las concentraciones de ácidos grasos monoinsaturados oscilaron entre 3,94 y $34.25 \%$, destacándose en Chacopata la alta concentración del ácido hexadecenoico (16:1) con un $28.83 \%$.
También, se obtuvieron otros ácidos grasos que no fueron identificados por la ausencia de sus patrones comerciales, los cuales oscilaron entre 17.84 y $46.82 \%$, pero por sus tiempos de retención, aparecieron en el rango de ácidos grasos insaturados del tipo poliinsaturados, se puede inferir que éstos son, en su mayoría, ácidos grasos insaturados.

\section{DISCUSIÓN}

Las diferencias en la concentración lipídica entre localidades, se pueden atribuir a varias causas, entre ellas: la disponibilidad de alimentos en las diferentes localidades, profundidad en la cual se reproduce la especie y los cambios bruscos de temperatura, $\mathrm{pH}$ y salinidad, entre otros. Las concentraciones más elevadas 
CUADRO 5

Composición porcentual de ácidos grasos de los triacilgliceroles de $\mathrm{P}$. viridis por área de estudio

TABLE 5

Percentage composition of fatty acids of $\mathrm{P}$. viridis triacylglycerols from the study areas

\begin{tabular}{|c|c|c|c|c|c|c|c|}
\hline Ácido graso & Río Caribe & La Restinga & La Esmeralda & Chacopata & La Brea & Los Cedros & Chaguaramas \\
\hline 11:0 & 3.45 & 22.59 & 1.67 & 0.88 & 0.69 & ------ & 10.04 \\
\hline 14:0 & 4.88 & 4.56 & 2.87 & ------ & 7.89 & 6.83 & 4.09 \\
\hline $16: 0$ & 12.59 & 13.05 & 14.36 & ----- & 14.67 & 5.42 & 10.48 \\
\hline $17: 0$ & 7.80 & 5.17 & 5.21 & ----- & 3.05 & 2.17 & 6.26 \\
\hline Total AGS & 42.37 & 53.80 & 41.21 & 14.27 & 40.41 & 32.83 & 42.62 \\
\hline $16: 1$ & 3.84 & 3.94 & 5.79 & 28.83 & 9.15 & ----- & 3.17 \\
\hline $18: 1$ & 0.54 & ----- & 1.75 & 2.28 & 2.49 & 4.06 & 0.83 \\
\hline Total AGMI & 4.38 & 3.94 & 9.49 & 34.25 & 13.78 & 5.90 & 3.99 \\
\hline $18: 3$ & ------ & 1.24 & 1.86 & 2.15 & 1.74 & 0.67 & ------ \\
\hline $18: 4$ & 5.39 & 2.90 & 1.47 & ------ & 2.72 & 3.44 & ------ \\
\hline $20: 4$ & ------ & ------ & 2.10 & 0.70 & ------ & ------ & ------ \\
\hline $20: 5 \omega-3$ & ------ & ----- & 1.49 & ----- & ----- & 7.64 & 1.72 \\
\hline $22: 4$ & 20.16 & 8.65 & 6.92 & ----- & 6.63 & 11.79 & 23.33 \\
\hline $22: 5 \omega-3$ & ------ & ----- & 6.36 & ----- & ----- & 4.87 & ------ \\
\hline $22: 6 \omega-3$ & ------ & 8.07 & ------ & 0.19 & ------ & ------ & ------ \\
\hline Total AGPI & 29.62 & 20.86 & 20.20 & 4.66 & 14.10 & 29.74 & 35.55 \\
\hline Total AGNI & 23.63 & 21.40 & 29.10 & 46.82 & 31.72 & 31.53 & 17.84 \\
\hline
\end{tabular}

$\mathrm{N}=3$, números de réplicas (pools de 60 mejillones); AGS=ácidos grasos saturados; AGMI=ácidos grasos monoinsaturados; AGPI=ácidos grasos poliinsaturados; AGNI=ácidos grasos no identificados; --------=no detectado; Valores encontrados $=($ medias \pm 0.1$)$; Límite de detección $\geq 0.1$.

en las muestras de Chaguaramas, La Brea y Los Cedros, pudo deberse a que las muestras tuvieron mejor ajuste de sus metabolismos a las condiciones ambientales de la zona.

Por otra parte, las concentraciones lipídicas reportadas en esta investigación son altas en comparación con estudios realizados en otras especies (D’Armas 1993, Malavé 1995, Milano 2003), esto se debe a que entre abrilagosto $P$. viridis se encuentra en condiciones de desove, lo que genera que tenga mayores reservas energéticas y, por lo tanto, mayor cantidad de lípidos (Shafee 1978). Con estos resultados se puede inferir, que las especies en periodo de maduración están íntimamente relacionadas con los cambios de las condiciones ambientales en cada época (sequía) y a la mayor o menor disposición de alimentos existentes en la zona de estudio para las especies, ya que algunas de ellas adquieren, en su dieta, alimentos diferentes. Durante la época de sequía existe la mayor disponibilidad de alimentos en el sistema acuífero (insectos, peces, vegetales, camarones y otros microorganismos) (Hellawell 1974).

Las muestras de Chacopata presentan la menor concentración de fosfolípidos, pero, a su vez, el mayor porcentaje de colesterol y triacilgliceroles. A pesar que en Chacopata, La Esmeralda y Río Caribe presentan niveles de temperatura y salinidad muy similares, los resultados obtenidos son contradictorios, esto puede atribuírsele a que las muestras provenientes de La Esmeralda y Río Caribe están cercanas a desagües de desechos fecales, lo que ocasiona gran cantidad de detritos (aproximadamente $75 \%$ ) y poca cantidad de fitoplancton, mientras que en Chacopata no existen descargas orgánicas. Además, esta área posee grandes 
oleajes, lo que pudiera originar la difícil adaptación de la especie y la poca capacidad para ajustar su metabolismo a esa condición, así como también un escaso contenido de sustancias nutritivas en sus aguas, desfavoreciendo así el engorde de los mejillones procedentes de esta área (Milano 2006).

Los bajos valores de fosfolípidos en las muestras de Chacopata pueden deberse a que éstos sufrieron degradación, los ácidos grasos se pierden por hidrólisis, causando una ruptura del enlace entre la base o el alcohol y el ácido fosfórico, haciendo que el $\alpha$-glicerol se hidrolice a glicerol y fosfato inorgánico, lo que puede ocasionar la síntesis de triacilgliceroles a partir de los ácidos grasos liberados, lo cual explicaría el mayor porcentaje de triacilgliceroles en las muestras de Chacopata. También, puedo deberse a que en esa área hubiese abundancia de fitoplancton y/o un patrón de circulación de corrientes marinas que favorezcan el crecimiento de la especie (Milano 2006).

Por otra parte, las elevadas concentraciones de fosfolípidos en las distintas muestras, se pueden atribuir a varias causas, entre los cuales se pueden sugerir que los fosfolípidos funcionan en algunos casos como catalizadores de oxidaciones biológicas, debido a la facilidad de autoxidación que sufren los ácidos grasos insaturados encontrados en su estructura (Devlin 1994). Otra causa que se le puede atribuir, es que los fosfolípidos en asociación con las proteínas y otros lípidos forman parte de los complejos lipoproteínicos que constituyen la matriz de las paredes de las membranas celulares (Ciarfella 1987); además, que la presencia de fosfolípidos puede ser debida a la hidrólisis de triacilgliceroles donde se forma 1,2-digliceroles a partir de los cuales es posible sintetizar fosfolípidos (Devlin 1994). También, las concentraciones de los fosfolípidos se pueden deber a los hábitos alimenticios de la especie en las diferentes zonas de estudio, ya que ellas se alimentan de fitoplancton, zooplancton, microalgas, entre otros y estas áreas son ricas en diversidad de estas especies. Al mismo tiempo, las variaciones de temperatura, salinidad, $\mathrm{pH}$, corrientes marinas y desarrollo de la especie influyen en las concentraciones de los diferentes tipos de lípidos, así como también pueden estar ligados a un acondicionamiento bioquímico de las mismas para la reproducción (Malavé 1995).

Los altos niveles de concentración de colesterol en las muestras de estudio, exceptuando las de las localidades de La Esmeralda y Río Caribe, pueden deberse a muchas causas como el que este lípido forma parte fundamental de la estructura de las membranas celulares de la especie en combinación con otros lípidos como los fosfolípidos y ésteres de colesterol, los cuales, probablemente, se relacionen con la regulación de la permeabilidad de las membranas celulares (Swinburn \& Ravussin 1993). Adicionalmente, en los tejidos animales, el colesterol es el precursor de otros muchos esteroides, como lo son los ácidos biliares, que ayudan a la emulsión de los lípidos y a su absorción intestinal (Leninger 1988). Así como también, las causas mencionadas anteriormente, como la alimentación de la especie y los ecosistemas donde habita dicha especie.

En los lípidos totales, las elevadas concentraciones de ácidos grasos saturados en las muestras de La Esmeralda, pueden atribuírsele al probable acondicionamiento energético de las gónadas para sus posteriores funciones reproductivas, esta localidad es muy explotada comercialmente y, en muchos casos, no se deja madurar a la especie en su totalidad. Estudios relacionados (Shafee 1978, Malavé 1995, Milano 2003) han demostrado que cuando la especie no ha alcanzado su madurez gonadal, la especie presenta concentraciones de ácidos grasos saturados superiores a los insaturados.

Además, los ácidos grasos poliinsaturados encontrados en los lípidos totales son de cadena muy larga, como el ácido eicosapentaenoico (20:5) y el ácido docosapentaenoico (22:5). Algunas investigaciones realizadas han establecido, que los ácidos altamente poliinsaturados provenientes de fuentes marinas pueden reducir los factores de riesgo relacionados a lesiones materiales como aterosclerosis y 
trombosis (Goldbourt \& Yaari 1990, D’Armas 1993).

Al comparar las concentraciones de ácidos grasos saturados obtenidos para los fosfolípidos y triacilgliceroles, se puede observar en todas las muestras, a excepción de los ácidos grasos encontrados en los triacilgliceroles de la muestra de Chacopata, que los valores de los ácidos grasos saturados fueron relativamente constantes, encontrándose los mismos ácidos grasos en las muestras estudiadas. Además diversas investigaciones sugieren que la elevada concentración de ácidos grasos saturados en $P$. viridis, está relacionado con los periodos de desove sexual, y se ha señalado como una posible causa de la degradación de los ácidos grasos poliinsaturados para la formación de eicosanoides, compuestos con una gran actividad fisiológica y farmacológica, como el caso de las prostaglandinas, las cuales tienen una participación importante en el crecimiento y reproducción de dichas especies (Malavé 1995).

En cuanto a los ácidos grasos insaturados se observó que éstos dominaron a los saturados en todas las localidades estudiadas. Dichos resultados coinciden con algunos autores que sugieren que las variaciones en los ácidos grasos poliinsaturados pueden deberse a que al ser componentes principales de los fosfolípidos y formar parte de las membranas, pueden ser usados como combustibles metabólicos. Además, señalan que la variabilidad de los ácidos grasos puede estar relacionada con los hábitos alimenticios de las especies marinas, así como con la disponibilidad de alimentos (fitoplancton) del medio (Gharseddiene 1999, D’Armas et al. 2010).

Además, la presencia de los ácidos grasos $20: 5 \omega-3,22: 5 \omega-3$ y $22: 6 \omega-3$, se justifica porque estos ácidos grasos se sintetizan a partir del ácido linolénico (18:3), mediante desaturaciones y elongaciones sucesivas. Estos ácidos grasos son esenciales para que la especie pueda cumplir con sus funciones reproductivas, asegurando el recurso energético y nutricional para el desarrollo y crecimiento de los nuevos seres; sin embargo, los seres marinos son incapaces de sintetizarlos, por tanto, su presencia en las diferentes muestras se debe a que los mismos son obtenibles a través de la dieta diaria (Villarroel 1997).

En este trabajo de investigación se logró determinar que el contenido de los fosfolípidos está en mayor proporción, seguido del colestrerol y luego los triacilgliceroles. La mayor concentración de ácidos grasos poliinsaturados se evidenció en las muestras de Chacopata y menor en La Esmeralda y Río Caribe. Además, el alto contenido de ácidos grasos insaturados encontrados en las muestras estudiadas, indica que el consumo frecuente del Perna viridis es recomendable, ya que podría ser beneficioso en la protección contra ciertas afecciones humanas como la obesidad, hiperlipidemia, cáncer, artritis y principalmente enfermedades cardiovasculares, disminuyendo así el riesgo a sufrirlas.

\section{AGRADECIMIENTOS}

Al Consejo de Investigación de la Universidad de Oriente y al Departamento de Química (Núcleo de Sucre) por haber financiado parcialmente esta investigación. A la Dra. Luisa Rojas y la Universidad de West Indies (Trinidad) por la recolección de las muestras.

\section{RESUMEN}

Los lípidos totales fueron extraídos, identificados y cuantificados por gravimetría, mediante TLC/FID (sistema Iatroscan). Los ácidos grasos de los lípidos totales, fosfolípidos y triacilgliceroles fueron identificados y cuantificados por cromatografía de gases. Las mayores concentraciones de lípidos totales se presentaron en las muestras de Los Cedros, La Brea y Chaguaramas con valores de 7.92, 7.74 y $7.53 \%$; respectivamente y los valores mínimos en las de La Restinga (6.08\%). En la composición lípidica, las muestras de Chacopata presentaron la menor concentración de fosfolípidos (48.86\%) y los máximos valores de colesterol (38.87\%) y triacilgliceroles (12.26\%), y las muestras de La Esmeralda y Río Caribe exhibieron concentraciones máximas de fosfolípidos (88.71-84.93\%, respectivamente) y mínimas de colesterol (6.50-4.42\%). En los lípidos totales, los ácidos grasos saturados se encontraron entre 15.04 y $65.55 \%$, con máximos en muestras de La Esmeralda y mínimos en Chacopata y los poliinsaturados estuvieron entre 7.80 y $37.18 \%$, con máximos valores en la Brea y mínimos en la Esmeralda. En los fosfolípidos, 
la concentración de ácidos grasos saturados estuvo entre 38.81 y $48.68 \%$ para las muestras de Chaguaramas y Chacopata, respectivamente; los ácidos grasos poliinsaturados se encontraron entre no detectado y $34.51 \%$, con altas concentraciones en muestras de Los Cedros (27.97\%) y Chaguaramas $(34.51 \%)$. En los triacilgliceroles, la composición de ácidos grasos saturados variaron entre 14.27 y $53.80 \%$, con baja concentración en Chacopata y alta concentración en La Restinga; los ácidos grasos poliinsaturados estuvieron entre 4.66 y $35.55 \%$, con valores menores en las muestras de Chacopata $(4.66 \%)$ y mayores en Chaguaramas. El consumo frecuente de Perna viridis es recomendable, debido a su alto contenido de fosfolípidos y ácidos grasos insaturados.

Palabras clave: ácidos grasos, bivalvo, lípidos, triacilgliceroles, Perna viridis.

\section{REFERENCIAS}

Ackman, R. 1982. Flame ionization detection applied to thin layer chromatography on coated quartz rods. Methods Enzymol. 72: 205-253.

Carter, L. 1993. Analysis of triglicerides. Academic, Nueva York, EEUU.

Ciarfella, A. 1987. Perfil lipídico en una muestra de la población Nor-Oriental de Venezuela. Trabajo de Pregrado, Departamento de Química, Universidad de Oriente, Cumaná, Venezuela.

Coristie, W. 1973. Lipids analysis. Pergamon, Nueva York, EEUU.

Cowey, C. \& J. Sargent. 1988. Nutrition. In W. Hoar \& J. Randall (eds.). Fish Physiology. Vol. 8. Academic, Nueva York, EEUU.

D’Armas, H. 1993. Aplicación de la resonancia magnética nuclear de c-13 y de técnicas cromatográficas al estudio de la composición lipídica de tejidos de las especies Hoplosternum littorale y Pseudoplastystoma fasciatum. Trabajo de Ascenso, Departamento de Química, Universidad de Oriente, Cumaná, Venezuela.

D’Armas, H., D. Yañez, D. Reyes \& G. Salazar. 2010. Composición de ácidos grasos de los caracoles marinos Phyllonotus pomum y Chicoreus brevifrons (Gastropoda: Muricidae). Rev. Biol. Trop. 58: 645-654.

Devlin, T. 1994. Bioquímica. Editorial Reverté, Barcelona, España.
Farnworth, E., B. Thompson \& J. Kramer. 1982. Quantitative determination of neutral lipids on chromarods. J. Chromatogr. 264: 463-747.

García, Y. 2003. Producción secundaria del Mejillón verde Perna viridis. Trabajo de Pregrado, Departamento de Biología, Universidad de Oriente, Cumaná, Venezuela.

Gharseddiene, S. 1999. Bioecología del isótopo litoral Tylos wegeneri (Vandel, 1952) (crustácea: isopoda) en Guayacán, estado Sucre, Venezuela. Tesis de Maestría, Universidad de Oriente, Núcleo de Sucre, Cumaná.

Goldbourt, V. \& S. Yaari. 1990. Cholesterol and coronary heart disease mortality. Aterosclerosis 10: 512-519.

Hellawell, J. 1974. The ecology of populations of dace Leuciscus leuciscus, from two tributaries of the River Wye. Freshw. Biol. 4: 577-604.

Lehninger, A. 1988. Bioquímica, las bases moleculares de la estructura y función celular. Editorial Omega, Barcelona, España.

Malavé, M. 1995. Caracterización y cuantificación del contenido lipídico y ácidos grasos presentes en dos especies de erizos de mar: Lytechinus variegatus y Echinometra lucunter. Trabajo de Pregrado, Universidad de Oriente, Cumaná, Venezuela.

Malavé, M. 2002. Caracterización y cuantificación del contenido lipídico presente en las gónadas y músculo de la especie de mejillón Perna perna. Trabajo de Ascenso, Universidad de Oriente, Cumaná, Venezuela.

Maza, A. 1991. Caracterización de lípidos en el tejido muscular de diferentes especies de peces de origen marino en el oriente del país. Trabajo de Pregrado. Departamento de Química, Universidad de Oriente, Cumaná. Venezuela.

Milano, J. 2003. Estudio comparativo del contenido lipídico, proteico y de glucógeno en la Crassostrea rhizophorae (ostra de mangles) provenientes de la Laguna de Chacopata, la Restinga y Caroní Swamp. Trabajo de Pregrado, Departamento de Química, Universidad de Oriente, Cumaná, Venezuela.

Milano, J, H. D`Armas \& G. Salazar. 2006. Perfil de ácidos grasos de Crassostrea rhizophorae proveniente de las lagunas de Chacopata y la Restinga (Venezuela) y el Pantano de Caroní (Trinidad y Tobago). Ciencias Marinas 32: 1-11.

Overturf, M \& R. Dryer 1967. Experiments in physiology and biochemistry. Academic, Nueva York, EEUU. 
Rojas, L. 2002. Chemical investigations on oysters and green mussels from Trinidad and Venezuela. Ph.D. Thesis, University of the West Indies, St. Augustine, Trinidad.

Shafee, M. 1978. Variations in biochemical composition of the green mussel Perna viridis Linnaeus of ennore estuary, Madras. Mah-Bull. of the Nat. Inst. of Oceanogr. 2: 101-104.

Swinburn, B. \& E. Ravussin. 1993. Energy balance or fat balance. Amer. Soc. Clin. Nutrit. 57: 766-771.
Vélez, A. \& R. Martínez. 1967. Reproducción y desarrollo larval experimental del mejillón comestible de Venezuela Perna perna (Linnaeus, 1758). Bol. Inst. Oceanogr. Univ. Oriente 6: 266-285.

Villarroel, A. 1997. Caracterización y cuantificación de las diferentes clases de lípidos presentes en el hígado y tejido muscular del Marao (Tylosurus crocodilus). Trabajo de Pregrado, Universidad de Oriente, Cumaná, Venezuela. 